\title{
CHAOS ASSISTED TUNNELING AND NONSPREADING WAVE PACKETS
}

\section{J. ZAKRZEWSKI}

Instytut Fizyki im. Mariana Smoluchowskiego, Uniwersytet Jagielloński

Reymonta 4, 30-059 Kraków*, Poland

\section{Delande}

Laboratoire Kastler-Brossel, Tour 12, Etage 1, Université Pierre et Marie Curie 4 Place Jussieu, 75-005 Paris, France

AND A. Buchleitner

Max-Planck-Institut für Quantenoptik Hans-Kopfermann-Str. 1, 85748 Garching, Germany

Consider an initial state lying on a primary resonance island. The state may tunnel into the chaotic sea surrounding it and further escape to infinity via chaotic diffusion. Properties of transport in such a situation are studied on an exemplary system - the hydrogen atom driven by microwaves. We show that the combination of tunneling followed by chaotic diffusion leads to peculiar large scale fluctuations of the AC Stark shift and ionization rates. An appropriate random matrix model describes accurately these statistical properties.

PACS numbers: $05.45 .+\mathrm{b}, 32.80 . \mathrm{Rm}, 42.50 . \mathrm{Hz}$

\section{Introduction}

Typical textbook cases of tunneling (in one-dimensional systems) consider quantum transport in situations when the classical transport between regions of space separated by e.g. potential barriers is forbidden. In multidimensional systems, the situation becomes complicated. Classically, in two-dimensions, Kolmogorov-Arnold-Moser (KAM) [1] tori provide strictly impenetrable borders for transport separating the phase space into distinct regions of classical motion ${ }^{\dagger}$. When the classical phase space is of the mixed type - partially chaotic and partially

*Permanent address.

†In higher-dimensional systems, classical Arnold diffusion provides another mechanism of classical transport, a process which is, however, typically very slow [1]. 
regular - composed of a chaotic sea and regular islands embedded in it, the classical transport between the islands and the sea is forbidden. Semiclassically, one can associate wave functions with distinct regions of phase space [2], the residual coupling between them will be due to quantum tunneling process. One can then consider the tunneling between two islands mediated by the classical transport in the chaotic sea surrounding them.

Interestingly, this chaos assisted tunneling mechanism possesses unique features typically absent in the standard "barrier" tunneling of quantum mechanics, such as a great sensitivity to the variation of external parameters manifesting itself in fluctuations of observable quantities. Previous works considered mainly model one-dimensional time dependent systems [3-5] or model two-dimensional autonomous systems [6-9]. A similar problem in the scattering case has also been discussed on a kicked model system [10].

We shall consider here a different situation, motivated by the physics of atoms ionized by external strong electromagnetic radiation. We discuss the single tunneling process out of the stable island followed by the chaotic diffusion process which eventually leads to ionization. The example studied, a hydrogen atom placed in the microwave field of circular polarization, is realistic and experimentally accessible.

Firstly we review the properties of states localized on stable primary islands - the so-called nonspreading wave packets. Later we concentrate on the properties of the decay of wave packet states showing, by comparison to a statistical model, that their mechanism of ionization is indeed due to chaos assisted tunneling process. The reader interested in details should consult the original papers [11-15].

\section{Nonspreading wave packets in periodically driven systems}

Consider a one-dimensional system described by the Hamiltonian $H_{0}(I)$ where $I$ is the principal action (we denote by $\varphi$ the angle conjugate to $I$ ). The frequency of the classical motion $\omega_{\mathrm{cl}}(I)=\partial H_{0} / \partial I$ is a function of the action and, therefore of the energy $E$. Now let us perturb the system by a time-periodic potential $V=v(I, \varphi) \cos (\omega t)$. If, for a given $E, \omega_{\mathrm{cl}}$ is vastly different from the external frequency $\omega$, the perturbation is nonresonant and affects only weakly the motion. The situation is drastically different in the case of a resonance, i.e. when $n \omega_{\mathrm{cl}}=m \omega$ with $n, m$ being low integers [1]. A resonant exchange of energy can take place, the system becomes strongly perturbed by $V$. Consider the simplest case of $1: 1$ resonance, i.e. when $\omega_{\mathrm{cl}} \approx \omega$. The Hamiltonian of the perturbed system can be (locally in energy) represented by the Hamiltonian of a pendulum with a pair of stable and unstable fixed points (periodic orbits of period $2 \pi / \omega$ ) [1].

While in the unperturbed system two initially close points $\left(I_{1}, \varphi_{1}\right)$ and $\left(I_{2}, \varphi_{2}\right)$ in the phase space tend to separate in angle from one another (since $\left.\omega_{\mathrm{cl}}\left(I_{1}\right) \neq \omega_{\mathrm{cl}}\left(I_{2}\right)\right)$ it may no longer be so for the perturbed motion. Inside the island surrounding the stable fixed point, the classical motion is restricted to tori surrounding the fixed point, the classical motion becomes locked to the external driving frequency.

Consider now a wave packet constructed for the unperturbed system. It will disperse following the fate of classical trajectories. By contrast, a wave packet placed inside the resonance island of the perturbed system will not disperse re- 
maining in the vicinity of the fixed point at every period of the external driving (following the corresponding periodic trajectory at all times).

Quantum mechanics of periodically perturbed systems tells us, by Floquet theorem [16], that the eigenstates of the system, the so-called quasi-energy states, are time periodic. If the classical island is large enough to support quantum states, some of the Floquet states must be localized inside the island. As exact solutions of the Schrödinger equation, they regain their shape every period and remain localized in the same region. Thus a quantum representation of the non-dispersive wave packet is simply a well chosen Floquet eigenstate.

First examples of Floquet states with such interesting properties were studied for model systems [17] and termed flotons. In an independent work, the non-dispersive wave packets have been semiclassically constructed for the hydrogen atom driven by a circularly polarized microwaves (CPM) [18]. Similarly such objects have been found for linearly polarized microwaves (LPM) [19] and identified with single Floquet eigenstates. For CPM this identification has been carried out in [11]. Let us mention also that the pendulum analysis in LPM case was also done [20]. Such a pendulum approach gives an excellent semiclassical prediction for the quasienergies of wave packet Floquet states.

For a hydrogen atom in CPM the situation is even simpler if not generic. In the frame rotating with the CPM field, the Hamiltonian becomes time independent

$$
H=\frac{p^{2}}{2}-\frac{1}{r}+F x-\omega \ell_{z}
$$

with $\ell_{z}$ the angular momentum operator. Atomic units are used throughout the paper.

At the center of the principal resonance island between the Kepler and the microwave frequency, a periodic orbit exists in the lab frame whose period exactly matches the period of the microwave. It corresponds to a fixed point of the motion in the rotating frame. It is possible to find the region of microwave fields when the point is stable [18], the semiclassical quantization based on a harmonic approximation around the fixed point yields accurate prediction for their energies [11]. Moreover, the wave packet is a coherent superposition of circular Rydberg states with a Gaussian-like distribution of the corresponding overlaps centered around the principal quantum number $n_{0}=\omega^{-1 / 3}$. Note that $n_{0}$ is not necessarily an integer.

Due to the scaling properties of the Coulomb problem, the classical dynamics depends only on the scaled microwave amplitude $F_{0}=F n_{0}^{4}=F \omega^{-4 / 3}$ which is the ratio of the microwave amplitude to the Coulomb field of the nucleus on the unperturbed $n_{0}$ circular orbit. The fixed point remains stable up to $F_{0} \approx 0.11$, i.e. fields which are strong enough to ionize a typical atomic state in few microwave periods [21]. In fact a significant ionization of a typical initial atomic state occurs already at $F_{0} \approx 0.03$ during few tens of microwave periods. Yet the lifetime against ionization of the wave packet states may well exceed million microwave periods! [12]. This is precise because these Floquet states are strongly localized on the stable island and classically forbidden to ionize. The spontaneous emission lifetime for the wave packets is exceedingly long [22], orders of magnitude longer than their lifetime against the ionization. Thus the ionization process is a 
dominant mechanism of the decay of wave packet states, the unusual properties of this decay are reviewed in the next section.

\section{Chaos assisted tunneling as a mechanism for ionization}

Using the expansion of Eq. (1) in the Sturmian basis combined with complex rotation technique and Lanczos diagonalization routine we are able to calculate exactly the quasienergy spectrum of the problem (for details and further references see $[12,15])$. The resulting Hamiltonian matrix is complex symmetric and yields eigenvalues of the form $\epsilon_{i}=E_{i}-\mathrm{i} \Gamma_{i} / 2$. For a bound state the imaginary part vanishes and $E_{i}$ is simply the energy of the state. For resonances, $E_{i}$ yields the energy position of the resonance while the corresponding $\Gamma_{i}$ is the resonance width. Excellent semiclassical prediction for the real part of the energy [11] allows us to extract only few eigenvalues (from matrices of a typical rank of 50000) around the semiclassical value, the wave packet state is then identified by its large dipole moment $\left(\approx n_{0}^{2}\right)$ in the rotating frame.

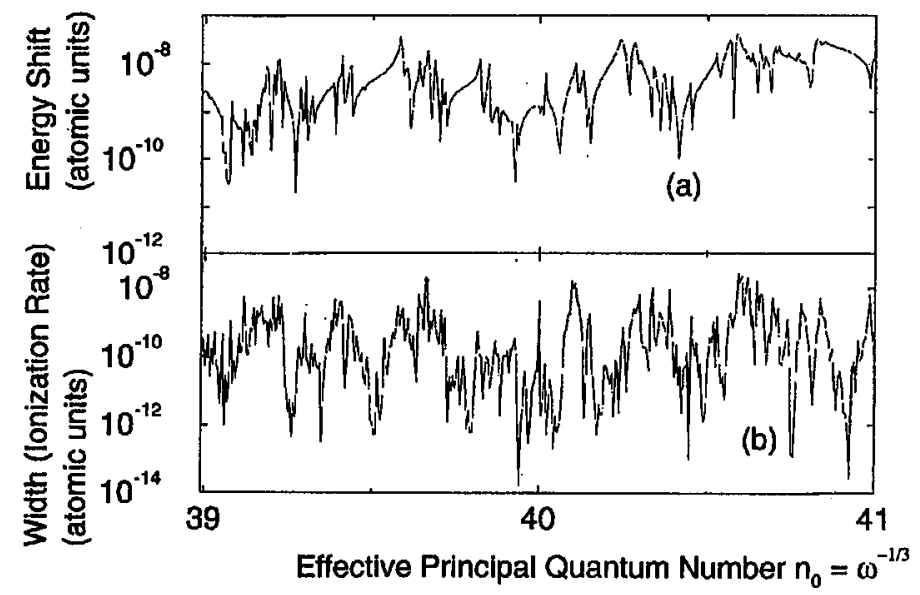

Fig. 1. Typical fluctuations of the width (ionization rate) (b) and the energy shift (a) (with respect to its unperturbed position) of the non-spreading wave packet of a hydrogen atom in CPM. The data presented are obtained for small variations of the effective principal quantum number $n_{0}$ around 40 , a scaled microwave electric field $F_{0}=F n_{0}^{4}=0.0426$ and a microwave frequency $\omega=1 / n_{0}^{3}$. To present both plots on the logarithmic scale, preferred to show the fluctuations over several orders of magnitude, we plot the absolute value of the shift rather than the shift itself.

The typical deviations of the exact resonance position from the semiclassical prediction and the ionization rates obtained are presented in Fig. 1. Observe the large scale fluctuations of both quantities over several orders of magnitude for small changes of the frequency (typically of the order of 1 part in 1000). These fluctuations - although perfectly deterministic - look completely random and are strongly reminiscent of the universal conductance fluctuations observed in mesoscopic systems [23]. Indeed, the ionization width measures the rate at which 
an electron initially localized close to the stable resonant trajectory ionizes, i.e. escapes to infinity. In other words, the ionization width directly measures the conductance of the atomic system from the initial point to infinity. In a quantum language, the ionization width is due to the coupling (via tunnel effect) between the localized wave packet and states lying in the chaotic sea surrounding it. While the energy of the wave packet is a smooth function of the parameters $F$ and $\omega$, the energies of the chaotic states display a complicated behavior characterized by level repulsion and strong avoided crossings. By chance, it may happen that - for specific values of the parameters - there is a quasi-degeneracy between the wave packet eigenstate and a chaotic state. There, the two states are more efficiently coupled by tunneling and the ionization width of the wave packet eigenstate increases. This is the very origin of the observed fluctuations. Simultaneously, the repulsion between the two states should slightly modify the energy (real part of the complex eigenvalue) of the wave packet state leading to fluctuations of the $\mathrm{AC}$ Stark shift.

To describe the fluctuations quantitatively we calculate the statistical distributions of the ionization widths $P(w)$ and of the energy shifts $P(s)$ and compare them with a simple statistical model [15]. The idea is to consider the wave packet eigenstate as coupled randomly with a set of chaotic states (described by random matrix theory [24]) themselves randomly coupled to the atomic continuum. For the detailed description of the model we refer the reader to [15]. In short the random realization of the Hamiltonian takes the form

$$
\mathcal{H}=\left(\begin{array}{cc}
E_{0} & \sigma V^{\mathrm{T}} \\
\sigma V & H_{0}-\mathrm{i} \gamma W W^{\mathrm{T}}
\end{array}\right),
$$

where $V$ is a random vector (whose components are Gaussian distributed random numbers) coupling a regular (wave packet) state with energy $E_{0}$ to $N$ chaotic states (eigenstates of $H_{0}$ ). The strength of the coupling is determined by $\sigma$. To model $H_{0}$ we use the standard assumption that $H_{0}$ pertains to the Gaussian orthogonal ensemble and generate $H_{0}$ accordingly [24]. The decay of chaotic states is due to a non-Hermitian part -i $\gamma W W^{\mathrm{T}}$ with strength determined by $\gamma . W$ itself is a random vector for a single channel decay [25]. The variance of the Gaussian distribution used to generate $H_{0}$ and the dimension of the matrix $N$ determine the mean level spacing $\Delta$ of the model. The two physically relevant, independent parameters are $\gamma / \Delta$ and $\sigma / \Delta$. In the perturbative regime $(\gamma / \Delta, \sigma / \Delta \ll 1)$ one may obtain analytically [15] the predictions for the distribution of shifts (of the regular state energy from the unperturbed value $\left.E_{0}\right) P(s)$ and that for the widths $P(w)$. Alternatively, as done here, one may find both distributions numerically by averaging over several realizations of random Hamiltonian Eq. (2). A comparison of the obtained distributions with data obtained for the real system - the hydrogen atom in CPM - allows us to extract the values of the physical parameters: $\sigma / \Delta$ - the coupling between the regular and chaotic states (the tunneling rate) and $\gamma / \Delta$ - the strength of the decay of chaotic states (the chaotic ionization rate). An example of such a fit is presented in Fig. 2. Typically around 1000 data points are taken around some mean value of $n_{0}$ and $F_{0}$. 


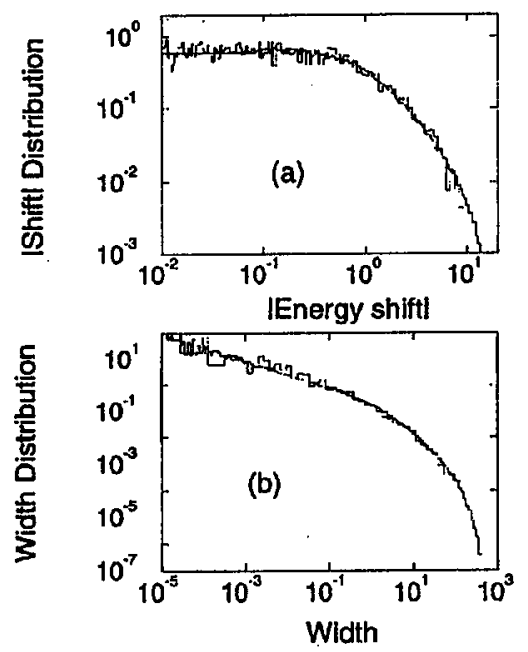

Fig. 2. The AC Stark shift distribution (a) and the width distribution (b) obtained from the data, part of which is shown in Fig. 1. Both distributions are shown in double logarithmic scale to better visualize the behavior over a range of shift and width values. Since AC Stark shift may be both positive and negative we show the distribution of the modulus. Large bin histograms correspond to hydrogen atom in circularly polarized microwaves, small bins to a fitted random model. Both distributions show the dominance of algebraic tail behavior followed by an exponential cut-off.

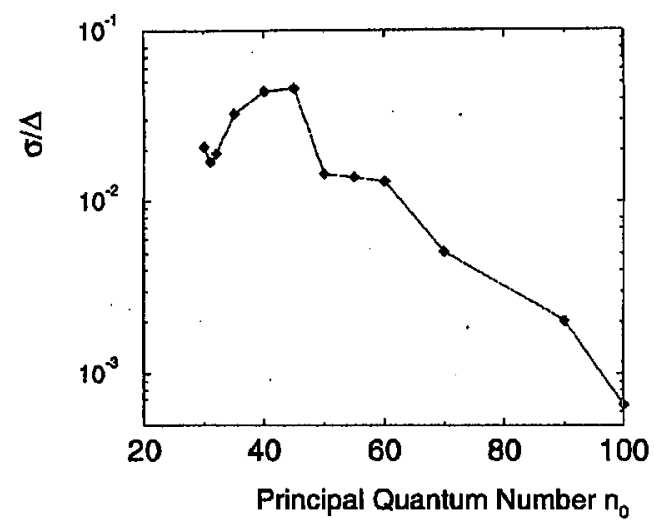

Fig. 3. The tunneling rate $\sigma / \Delta$ as a function of the effective quantum number $n_{0} \propto 1 / \hbar$ for a fixed classical dynamics, $F_{0}=0.0426$. Note the exponential decrease for sufficiently high $n_{0}$ (vertical scale is logarithmic).

Taking several stretches of data corresponding to different mean $n_{0}$ and the same $F_{0}$ one can test the $\hbar$ dependence of the tunneling rate and the ionization rate. The effective $\hbar$ in our problem is inversely proportional to $n_{0}$. It turns out that $\gamma / \Delta$, the chaotic ionization rate, is only weakly dependent on $n_{0}$. This is 
understandable - the ionization of chaotic states originates from the classical diffusion. However, as depicted in Fig. 3, $\sigma / \Delta$, i.e., the tunneling rate decays exponentially with $n_{0}$ to a good accuracy. Writing the tunneling rate as $\sigma / \Delta \propto$ $\exp (-S / \hbar) \propto \exp \left(-S n_{0}\right)$ we can extract by a straight line fit, the tunneling action value $S=0.06 \pm 0.01$. This exponential behavior is a strong evidence of the tunneling mechanism of the ionization.

\section{Conclusions}

We have provided a numerical evidence for a novel mechanism of ionization - chaos assisted tunneling - by studying a realistic and experimentally accessible example - a hydrogen atom driven by circularly polarized microwaves. It turns out that both the widths and AC Stark shifts of nonspreading wave packet states exhibit large scale fluctuations. We analyse these fluctuations quantitatively using an appropriately defined random matrix model. The model allows us to separate the ionization into two stages: tunneling from a stable island into the surrounding chaotic sea (which shows the typical exponential dependence on $\hbar$ ) and the chaotic diffusion process (weakly dependent on $\hbar$ ) which leads to further excitation and finally ionization.

\section{Acknowledgments}

CPU time on a Cray C98 computer has been provided by IDRIS. Laboratoire Kastler Brossel de l'Université Pierre et Marie Curie et de l'Ecole Normale Supérieure is unité associée 18 du CNRS. J.Z. acknowledges support of the Committee for Scientific Research (Poland) under project No. 2P03B 03810. The additional support under the bilateral collaboration scheme (J.Z. and D.D.) of the French Embassy in Poland, no. 76209 and the Programme International de Coopération Scientifique (CNRS) no. 408 is appreciated.

\section{References}

[1] A.J. Lichtenberg, M.A. Liberman, Regular and Chaotic Dynamics, Springer-Verlag, New York 1992.

[2] I.C. Percival, J. Phys. B 6, L229 (1973).

[3] W.A. Lin, L.E. Ballentine, Phys. Rev. Lett. 65, 2927 (1990); Phys. Rev. A 45, 3637 (1992).

[4] F. Grossmann, T. Dittrich, P. Jung, P. Hänggi, Phys. Rev. Lett. 67, 516 (1991);

Z. Phys. B 84, 315 (1991); J. Stat. Phys. 70, 229 (1993).

[5] J. Plata, J.M. Gomez Llorente, J. Phys. A 25, L303 (1992).

[6] O. Bohigas, S. Tomsovic, D. Ullmo, Phys. Rep. 223, 43 (1993).

[7] O. Bohigas, D. Boosé, R. Egydio de Carvalho, V. Marvulle, Nucl. Phys. A 560, 197 (1993).

[8] S. Tomsovic, D. Ullmo, Phys. Rev. E 50, 145 (1994).

[9] F. Leyvraz, D. Ullmo, J. Phys. A 29, 2529 (1996).

[10] P. Gerwinski, P. Seba, Phys. Rev. E 50, 3615 (1994).

[11] D. Delande, J. Zakrzewski, A. Buchleitner, Europhys. Lett. 32, 107 (1995). 
[12] J. Zakrzewski, D. Delande, A. Buchleitner, Phys. Rev. Lett. 75, 4015 (1995).

[13] J. Zakrzewski, A. Buchleitner, D. Delande, Z. Phys. B 103, 115 (1997).

[14] J. Zakrzewski, D. Delande, J. Phys. B, Atom. Mol. Opt. Phys. 30, L87 (1997).

[15] J. Zakrzewski, D. Delande, A. Buchleitner, unpublished.

[16] J.H. Shirley, Phys. Rev. 138, B979 (1965).

[17] J. Henkel, M. Holthaus, Phys. Rev. A 45, 1978 (1992); M. Holthaus, Chaos Solitons Fractals 5, 1143 (1995).

[18] I. Białynicki-Birula, M. Kaliński, J.H. Eberly, Phys, Rev. Lett. 73, 1777 (1994).

[19] A. Buchleitner, thèse de doctorat, Université Pierre et Marie Curie, Paris 1993, unpublished; D. Delande, A. Buchleitner, Adv. At. Mol. Opt. Phys. 35, 85 (1994); A. Buchleitner, D. Delande, Chaos Solitons Fractals 5, 1125 (1995); Phys. Rev. Lett. 75, 1487 (1995).

[20] L. Sirko, P.M. Koch, Appl. Phys. B 60, 195 (1995).

[21] J. Zakrzewski, R. Gębarowski, D. Delande, Phys. Rev. A 54, 691 (1996).

[22] Z. Białynicka-Birula, I. Białynicki-Birula, submitted to Phys. Rev. A; K. Hornberger, A. Buchleitner, submitted to Phys. Rev. Lett.

[23] S. Washburn, R. Webb, Adv. Phys. 35, 375 (1986); D. Mailly, M. Sanquer, J. Phys. I (France) 2, 357 (1992); P.A. Lee, A.D. Stone, Phys. Rev. Lett. 55, 1622 (1985).

[24] O. Bohigas, in: Chaos and Quantum Physics, Eds. M.-J. Giannoni, A. Voros, J. Zinn-Justin, Les Houches Summer School, Session LII, North-Holland, Amsterdam 1991, p. 87.

[25] F. Haake, F. Izrailev, N. Lehmann, D. Saher, H.-J. Sommers, Z. Phys. B 88, 359 (1992). 